\title{
LOS NUEVOS YACIMIENTOS Y LA CREACIÓN DE EMPLEO EN LA ECONOMÍA ANDALUZA: IMPLICACIONES PARA LOS MERCADOS DE TRABAJO LOCALES*
}

\author{
José Luis Martín Navarro \\ Luis Palma Martos \\ Universidad de Sevilla
}

Los Nuevos Yacimientos de Empleo (NYE) surgen a partir del libro blanco Crecimiento, competitividad y empleo, publicado en 1993 como un concepto novedoso que puede contribuir a la reducción de las altas tasas de desempleo que soporta en esta década el conjunto de la Unión Europea. Andalucía es una de las regiones con tasa de paro más alta y es en ella donde centramos nuestro análisis de los NYE. El presente trabajo trata de de ofrecer una visión de conjunto de los NYE, de sus fortalezas y debilidades como instrumento efidaz para crear empleo Planteamos un modelo simple que pretende servir de reflexión sobre su potencialidad para crear empleo en mercados de trabajo locales. Por último se plantea un análisis más general sobre la posibilidad de aplicación del concepto al conjunto de la economía andaluza.

The New Sources and creation of employment in the Andalousian economy: implications for local labour markets.

The idea of the New Sources of Employment (NSE) appears in the white book, Growth, Competitiveness and Employment, published in 1993, as a new concept for helping to reduce the high rates of unemployment that characterize the European Union in that year. Andalusia is at the moment one of the European regions with a higher rate. We focus the analysis on this particular region. A general vision of

- Este artículo, aunque planteado en clave teórica, tiene su origen en dos Proyectos de Investigación. El primero de ellos, por razón de la cronología, titulado "Estrategias territoriales de creación de actividades" fue financiado por la Dirección General V de la Comisión de las Comunidades Europeas. El segundo, bajo el título "Análisis metodológico para la evaluación de yacimientos de empleo: una aplicación a Sevilla y su provincia", ha sido financiado por el Instituto de Estadistica de Andalucía. Agradecemos a ambas instituciones su apoyo. 
the NSE, its weaknesses and fortresses as an effective instrument for the creation of employment, is offered in this paper.

We describe a simple model that may be useful to analyse the potentiality of NSE for the cri.lliun of new employment in local labour markets. Finally. we offer a general analysis about the possibility of the application of this concept to the Andalousian economy.

Palabras clave: Nuevos Yacimientos de Empleo. Paro. Economia Andaluza. Desarrollo Local.

Keyurords: Neu: Sources of Employment. unemployment, andalousian economy. local development.

\section{INTRODUCCIÓN}

La publicación en Diciembre de 1993 del Libro Blanco Crecimiento, Competitividad, Empleo retos y Pistas para entrar en el siglo XXI supone un hito en la redefinición política del problema del desempleo en la Unión Europea. Al menos en lo que se refiere a la enunciación de principios.

Entre 1980 y 1992 la tasa media de desempleo en la Unión Europea había pasado del $6,2 \%$ al $9.8 \%$, si bien países como España alcanzaban la desorbitada cifra del 18,1\%. Aún crecería el desempleo en Europa, puesto que en 1996 la cifra media llegó al $10 \%$ y en el caso español se situó en el $22,5 \%$. En este sentido, el Libro Blanco de 1993 supuso una voz de alerta contundente'.

En realidad. la Unión Europea estaba inmersa en la fecha de aparición del Libro Blanco en otro combate, al que se le estaba dando prioridad, la lucha contra la inestabilidad de precios. La declaración de guerra contra el desempleo se formula, pero se aplaza la acción hasta concluir una campaña dificil como era el proceso de convergencia hacia la moneda única, culminado en Mayo de 1998. No puede afirmarse que la dejación respecto a la lucha contra el desempleo fuese total, pero las prioridades han estado claras. Una vez alcanzada con éxito la solución al problema prioritario, o al menos una vez encauzada la política económica europea en la senda marcada por el Programa de Convergencia consensuado en Maastricht, se hace necesario atender al problema del desempleo por pura prudencia política en aras al mantenimiento de la concordia y la cohesión social, características de Europa desde la finalización de la Segunda Guerra Mundial.

A las bases programáticas aparecidas en el Libro Blanco se unen en 1996 las recogidas en el documento de la Comisión de las Comunidades Europeas titulado ACCIÓN A FAVOR DEL EMPLEO EN EUROPA. PACTO DE CONFLANZA, que en su primer párrafo señala:

1. Su primer párrafo, al justificar el por qué de la redacción del libro dice: "La razón es una sola. Una palabra: el paro. Conocemos su magnitud y también sus consecuencias. Y la experiencia demuestra lo arduo que resulta combatirlo". 
Desde la década de 1970. el paro es endémico en Europa. Además asistimos a un aumento regular del indice de desempleo estructural. Después de los periodos de recesión los niveles de paro son cada vez mâs altos... Frente a esta siluación, es preciso reaccionar. La persistencia del paro debilita la sociedad y desgasta a millones de hombres y mujeres. Hay que establecer el clima de confianza en Europa, como condición previa de la recuperación de la inversión y del consumo.

Este párrafo resume con precisión la naturaleza y consecuencias del desempleo y alerta sobre la necesidad de buscar soluciones urgentes².

A finales de 1998 las cifras de desempleo habían mejorado. La cifra para la economía española se situaba en el 18,17\%, y Andalucía presentaba una tasa del 29,27\%. Las tasas de desempleo bajan, pero los diferenciales se mantienen en los ya persistentes 10\% entre Europa y España y otro diez entre España y Andalucía. Menos paro pero no más convergencia en términos de empleo.

Por otra parte, en la cumbre de Iuxemburgo, celebrada en Noviembre de 1997 se plantearon tímidamente posibles vias para establecer un Programa de Convergencia por el empleo, pero desde entonces no se ha avanzado decididamente en esta dirección, salvo en la definición de los planes de empleo de cada país.

En este contexto general de Convergencia Europea para la creación de la Unión Económica y Monetaria y de la declaración de intenciones sobre la necesidad de crear empleo surge un nuevo concepto. Los llamados yacimientos de empleo, procedentes del Libro Blanco de 1993, se han convertido en la vía más estudiada, de todas las planteadas recientemente para la creación de nuevo empleo. Pero quizás lo ha sido, en buena medida, con una carencia de tono crítico, y una cierta utilización generalizada y poco científica. Sin embargo, este concepto significa un importante cambio en el enfoque del análisis: de lo macro a lo micro y de lo nacional a lo regional o lo local. Quizás una nueva manera de entender el desarrollo económico y la creación de empleo.

El trabajo que introducimos con estas líneas trata de ofrecer una visión del fenómeno de los yacimientos de empleo referida especialmente a la economía regional de Andalucía. En el siguiente epígrafe plantearemos de forma general las características de los Nuevos Yacimientos, así como sus fortalezas y debilidades como instrumento para la creación de empleo. A continuación se presenta un modelo simple que integra el fenómeno de los yacimientos al objeto de analizar sus implicaciones en un mercado de trabajo local. Por último, se señala la posible incidencia de esta nueva orientación política sobre el empleo en el mercado de trabajo andaluz.

2. En DÁVILA MURO, G. (1997) podemos seguir la trayectoria histórica de la nueva estrategia económica en la lucha contra el desempleo desde la publicación del Libro Blanco en 1993 hasta el Consejo Europeo de Dublín de diciembre de 1996. 


\section{LOS NUEVOS YACIMIENTOS Y LA CREACIÓN DE EMPLEO}

Es un principio que se está imponiendo gradualmente que las políticas tradicionales contra el desempleo carecen de eficacia en el entorno actual. El ya citado Libro Blanco de 1993 introduce nuevas líneas para la detección y explotación de fuentes de empleo y ahí se enmarca el concepto de nuevos yacimientos de empleo.

Como indicamos en la introducción, este documento supone la primera manifestación clara de preocupación por el problema del desempleo que hace la Unión Europea. El informe es ciertamente ambicioso. Determina un marco global de acción, que teniendo en cuenta las peculiaridades de cada país miembro, permita adoptar una línea de actuación común para la lucha contra el paro.

En el estudio, se realiza un análisis de la situación y perspectivas de las economías europeas. En este sentido, y en términos generales, los elevados niveles de desempleo se deben a dos causas principales. De una parte, se puede decir que ha existido una mala gestión macroeconómica de la economía, pues los instrumentos utilizados tradicionalmente han sido agotados o ya no son capaces de generar nuevos efectos en la economía a la que se aplican y además han sido mal usados en algunas ocasiones.

Por otra parte, no ha existido una suficiente adaptación a los cambios habidos tanto en la estructura económica europea como en la situación internacional. La estructura productiva no se adecua a las nuevas condiciones, ya que es reflejo de la situación existente cuando se crea dicha estructura: mano de obra escasa, recursos naturales que no presentan restricciones de utilización y evolución tecnológica asimilable por la propia dinámica de la economía. Esta falta de adecuación provoca en la actualidad un alto desempleo estructural y tecnológico que además contribuye al mantenimiento de altas tasas de desempleo de larga duración.

Con especial relación al desempleo tecnológico señala MARTín (1997, pp. 7778):

Una proporción no despreciable del paro se debe a las limitaciones existentes en los sistemas tecnológicos y educativo de los países para aprovechar adecuadamente el potencial de las numerosas innovaciones técnicas que se ban venido generando en los ültimos años en diversas áreas.

Además de todo ello, el entorno macroeconómico en el que se desenvuelve la economía europea para potenciar el incremento del empleo en los últimos años se define por una serie de características muy específicas: estabilidad monetaria y cambiaria, estabilidad de precios y un necesario crecimiento económico que sea capaz de generar empleo. Este nuevo escenario determina unas estrictas condiciones de 
estabilidad en el sentido de los criterios de convergencia de Maastricht, dejando poco margen de maniobra a las tradicionales políticas económicas y exigiendo nuevas ideas y lineas de actuación a los gobernantes.

El escenario en el que deben plantearse nuevas vías de solución viene caracterizado por unos elementos comunes a todas las economias occidentales. En concreto podemos señalar:

1. El moderado crecimiento del empleo está asociado con unas tasas muy elevadas de crecimiento de la producción.

2. Una tasa de actividad que para la Unión Europea no se ha visto aumentada esencialmente desde 1980 (se encuentra en torno al 66.8\% $\%^{5}$ ). Así mismo, se da una asimetría en el reparto de esta tasa entre hombres y mujeres, en perjuicio de estas últimas.

3. La estructura del desempleo por edades dista de ser homogénea, acusando una mayor incidencia entre los más jóvenes y los trabajadores que han superado los cuarenta años.

4. La participación de los sectores productivos se ha alterado significativamente en detrimento del sector primario y en un claro beneficio del sector servicios.

Para el caso español, una serie de perturbaciones de oferta y demanda ha magnificado los aspectos distorsionadores de algunas instituciones del mercado de trabajo. Entre ellos podemos señalar en primer lugar, un sistema de contratos mal diseñado y reformado dos veces en fechas recientes (1994 y 1997), que ha determinado una segmentación del mercado entre trabajadores fijos y temporales. En segundo lugar, el sistema de protección al desempleo, con claras consecuencias desincentivadoras a la búsqueda de empleo y que favorece la alta tasa de paro de larga duración (JIMENO, J. F. 1998). Además, se pueden incluir entre las características peculiares del mercado de trabajo español que han contribuido, en diversa medida, a la alta tasa de desempleo, el sistema de negociación colectiva, la estructura sindical, el sistema de despidos y regulación de plantillas o los costes laborales indirectos.

La problemática presentada para la economía española se acentúa en el caso de Andalucía. La economía andaluza muestra rasgos de especialización productiva en sectores en recesión como es el caso de la Agricultura, y el sector industrial tiene un peso relativo bastante bajo. Además, la tasa de actividad es menor que la espanoola y las tasas de desempleo juvenil y de larga duración, mayores, así como la tasa total de paro. Estas características negativas del mercado laboral deben combi-

3. Datos de la OCDE para 1996. 
narse con una clara debilidad de la economía andaluza. Manifestaciones de esta debilidad serían el nivel de renta (un $60 \%$ de la media europea), una endeble y mal articulada estructura-productiva, un gasto en $I+D$ por debajo de la media nacional (que ya está más de un punto por debajo de la media comunitaria) y la carencia de un tejido empresarial de calidad. Todos estos aspectos hacen que la situación en el mercado de trabajo andaluz sea bastante crítica y se hacen necesarias medidas urgentes y capaces de abordar con profundidad la cuestión.

En definitiva, como señala TOHARÍA (1998): Parece claro que la economía española necesita crear mucho empleo si quiere absorber en un plazo razonable su enorme volumen de paro. En este sentido, las observaciones del "Libro Blanco" de la Comisión Europea relativas a la existencia de muchas demandas insatisfechas en las sociedades modernas se vuelven absolutamente relevantes y las posibilidades de aprovechar ese potencial descle el punto de vista del empleo fundamentales.

En este contexto, es en el que se encuadra la aparición de los Nuevos Yacimientos de Empleo. Los objetivos básicos que se pretenden alcanzar con ellos son dos. Por un lado, la mejora de la competitividad de la economía europea, y por otro elevar el bienestar de la población. La consecuencia de estos objetivos exige delimitar un ámbito privilegiado para la intervención de los agentes. A las clásicas iniciativas, pública y privada, debe unírseles la de carácter asociativo.

Las nuevas necesidades que sustentan las nuevas fuentes de empleo proceden de las transformaciones, tanto de indole demográfica como social, que han sufrido las sociedades occidentales en las tres últimas décadas y que se vienen acentuando en la actualidad. Se citan de modo recurrente, el progresivo envejecimiento de la población, la profunda transformación de la estructura familiar y el mayor acceso de la mujer al mercado de trabajo. Habría que citar también el relevante cambio habido en el uso del tiempo y el espacio. Es una evidencia significativa la tendencia a la reducción de la jornada de trabajo y el consecuente aumento de la demanda de actividades de ocio. Podría destacarse también la modificación en los hábitos de consumo, consecuencia de las innovaciones en los campos de la electrónica, la informática o las comunicaciones.

Todas estas transformaciones han generado un cambio sustancial en las necesidades tanto personales como sociales. Estos cambios afectan, como hemos señalado, a la vida en el hogar, la ocupación del tiempo libre, la demanda de una mayor calidad de vida o la definición actualizada de la solidaridad entre generaciones.

Los nuevos yacimientos de empleo tienen su origen en la posibilidad de que estas necesidades individuales y sociales adquieran la condición de demandas efectivas. Aunque el sector servicios aparece como el de mayor potencialidad, estas nuevas fuentes de empleo abarcan todos los sectores productivos.

La Comisión Europea, en una encuesta llevada a cabo en 1995, señala cuatro ámbitos que recogen un total de 17 yacimientos de empleo. La clasificación sería la 
siguiente: 1) Servicios de la vida cotidiana (servicios a domicilio, atención a la infancia y las personas mayores); 2) Servicios de mejora de la calidad de vida (mejora de las viviendas, seguridad, transportes colectivos locales, comercios de proximidad); 3) Servicios de ocio (turismo, audiovisual, valorización del patrimonio cultural, deporte); 4) Servicios medioambientales (gestión de residuos, gestión del agua, protección y mantenimiento de zonas naturales, control de la polución).

Estas fuentes de creación de empleo suponen satisfacer necesidades generadas en un ámbito cercano al ciudadano, de ahí lo trascendente de cualquier análisis al respecto para las decisiones políticas, individuales o sociales en el ámbito localt.

\section{CARACTERIZACIÓN Y METOdología de INTERVENCión}

Según indican COLLADO y MARTÍNEZ (1995), la aproximación a los nuevos yacimientos de empleo desde una perspectiva económica se puede hacer aplicando los elementos de análisis que se asocian a los mercados especiales que se caracterizan por ser incompletos, inexistentes o con dificultades de identificación de los costes.

Los nuevos yacimientos de empleo tienen características específicas asociables a mercados imperfectos con ámbitos de producción y prestación definidos. Así mismo, se caracterizan por ser intensivos en factor trabajo. Otro elemento a considerar es la contradicción que late al ser el empleo, como bien social, el núcleo del análisis. Su generación resulta costosa, pues la mano de obra es un factor de producción de coste elevado. Así, la propia naturalezáa intensiva en mano de obra de estas fuentes de empleo lleva implícita su debilidad al suponer procesos de producción caros.

En síntesis, y siguiendo a JIMENEZ et al. (1998) la definición de una actividad como yacimiento de empleo debe integrar cuatro características: 1) Estar destinadas a satisfacer nuevas necesidades sociales; 2) que se configuren como mercados incompletos; 3) que tengan un ámbito de producción/prestación territorial definido y 4) que sean intensivas en empleo.

Estas características dificultan el desarrollo de las actividades. Los mercados incompletos presentan dificultades en la especificación del servicio a prestar, así como de la demanda existente, pues ésta puede resultar invisible y con problemas de solvencia presupuestaria para su materialización. La oferta se caracteriza por ser fragmentaria, de baja calidad y con sustitutivos cercanos fuera del mercado. Pueden señalarse, además, dos características específicas. En primer lugar, la invisibilidad

4. Véase el trabajo realizado para definir, en base a estos yacimientos, los empleos de futuro en la provincia de Sevilla, UGT (1998). 
del coste y por consiguienté la ausencia de un precio único. En segundo lugar, es muy difícil la introducción de elementos para la mejora de la productividad, dada la naturaleza intensiva en factor trabajo de estas actividades.

En resumen, son mercados con alta interdependencia entre la oferta y la demanda, sometidos a una fuerte competencia de mercados paralelos e incluso de mercados irregulares, lo que supone la existencia de abundantes sustitutivos tanto en la esfera del mercado como en la del no-mercado.

Para plantear el análisis de intervención sobre este tipo de mercados podemos seguir a JIMENEZ et al. (1998). Consideremos simplificadamente que los bienes que se analizan tiene las características de bienes públicos. Se plantea el objetivo de la intervención en el mercado para expandir la demanda y mejorar la calidad y cantidad de la oferta. En la Figura 1 podemos observar la demanda $D_{1}$, típica de bien público. Los consumidores deben pagar un precio igual al coste marginal de producción. Se observa una alta elasticidad a medida que los precios bajan. Para el precio $\mathrm{p}_{1}$ el volumen demandado es escaso, $\mathrm{q}_{1}$, y por tanto la oferta pública de ese bien es también pequeña.

En este mercado la intervención pública trata de expandir la demanda, por ejemplo mediante la concienciación de los ciudadanos, potenciales usuarios, de que es precisa su colaboración para cubrir los costes de producción, así como de los efectos beneficiosos, individual y socialmente, del consumo del bien o servicio.

Figura 1

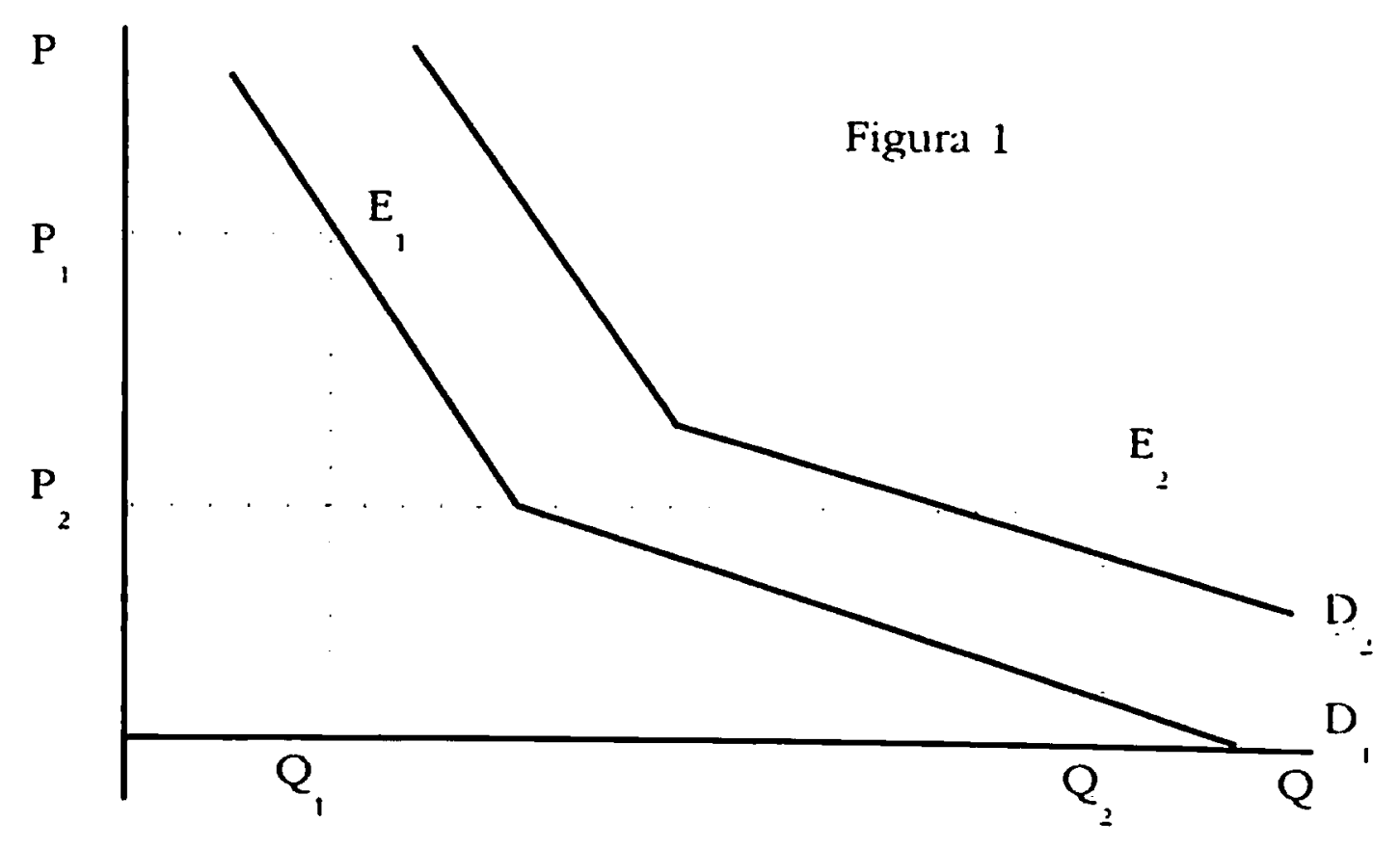


Junto con esta concienciación ciudadana es aconsejable una acción pública que mejore la productividad y que incentive el consumo a base de menores precios que no obstante, sigan cubriendo los costes. Vemos como el nuevo equilibrio, $\mathrm{E}_{2}$. sobre la nueva curva de demanda, $\mathrm{D}_{2}$, supone un sustancial aumento del consumo del bien o servicio, así como un abataramiento del mismo. De esta forma sencilla, podemos ilustrar la manera en que se puede incidir sobre un tipo de empleo que queremos potenciar. Pero antes de una intervención pública, debemos medir si el yacimiento de empleo existe y de qué cantidad se trata.

Por ello, más allá de caracterizar la naturaleza de estas nuevas fuentes de empleo es preciso aproximar una metodología que nos permita una evaluación del yacimiento en términos cuantitativos y sirva de guía para la intervención pública que acabamos de modelizar en la Figura 1. Vamos a plantear una metodología en cuatro fases que nos permitirá una primera evaluación del yacimiento. Posteriores estudios, más precisos, nos podrían ofrecer un mayor detalle, pero nos interesa una visión previa que oriente decisiones políticas de apoyo en una u otra dirección

\section{- Primera fase: Evaluación de la demanda potencial}

Tras identificar una necesidad insatisfecha para una población que habita en un área geográfica delimitada (Población de referencia), debemos calcular el número de individuos de dicha población de referencia que realmente manifiestan sentir esta necesidad, al que denominamos universalidad. También es preciso conocer la intensidad con la que la población de referencia siente estas necesidades, así como los factores determinantes de esa intensidad.

Puede resultar de interés en esta fase disponer de encuestas o estudios de mercado que especifiquen los grados de intensidad sentidos así como la universalidad de cada necesidad. Aquí se incluye el efecto de la renta y la disponibilidad a pagar para satisfacer la necesidad.

\section{- Segunda fase: Evaluación de la oferta disponible}

Conocida la demanda potencial se analiza la oferta disponible en los ámbitos público, privado y en la economía social.

- Tercera fase: Por diferencia, conocemos la demanda insatisfecha.

5. Esta metodología se ha diseñado y aplicado en un proyecto europeo bajo el título "Estrategias territoriales de creación de actividades" financiado por la Dirección General V de la Comunidad. Fn el proyecto han participado las Universidades de Patras, Génova, Berlín, Collège Coopératif de Aix en Provençe y Sevilla, así mismo los ayuntamientos de las respectivas ciudades. En el caso del Collège Coopératif participó el ayuntamiento de Marsella. Los firmantes de este artículo participaron como investigadores en el equipo de la Universidad de Sevilla. 
- Cuarta fase: Cuantificación de los puestos de trabajo a crear. Usualmente se calculan las horas anuales necesarias para la provisión de los bienes o servicios que componen la demanda insatisfecha. Así, el yacimiento queda evaluado en términos de puestos de trabajo a tiempo completo -8 horas/día- a partir de estas horas anuales en total.

Finalmente, se debería plantear un análisis de viabilidad de la actividad propuesta, en el que se estime la inversión necesaria para la creación de los puestos de trabajo.

En la Figura 2 se plantea de un modo sintético el proceso expresado más arriba.

Figura 2
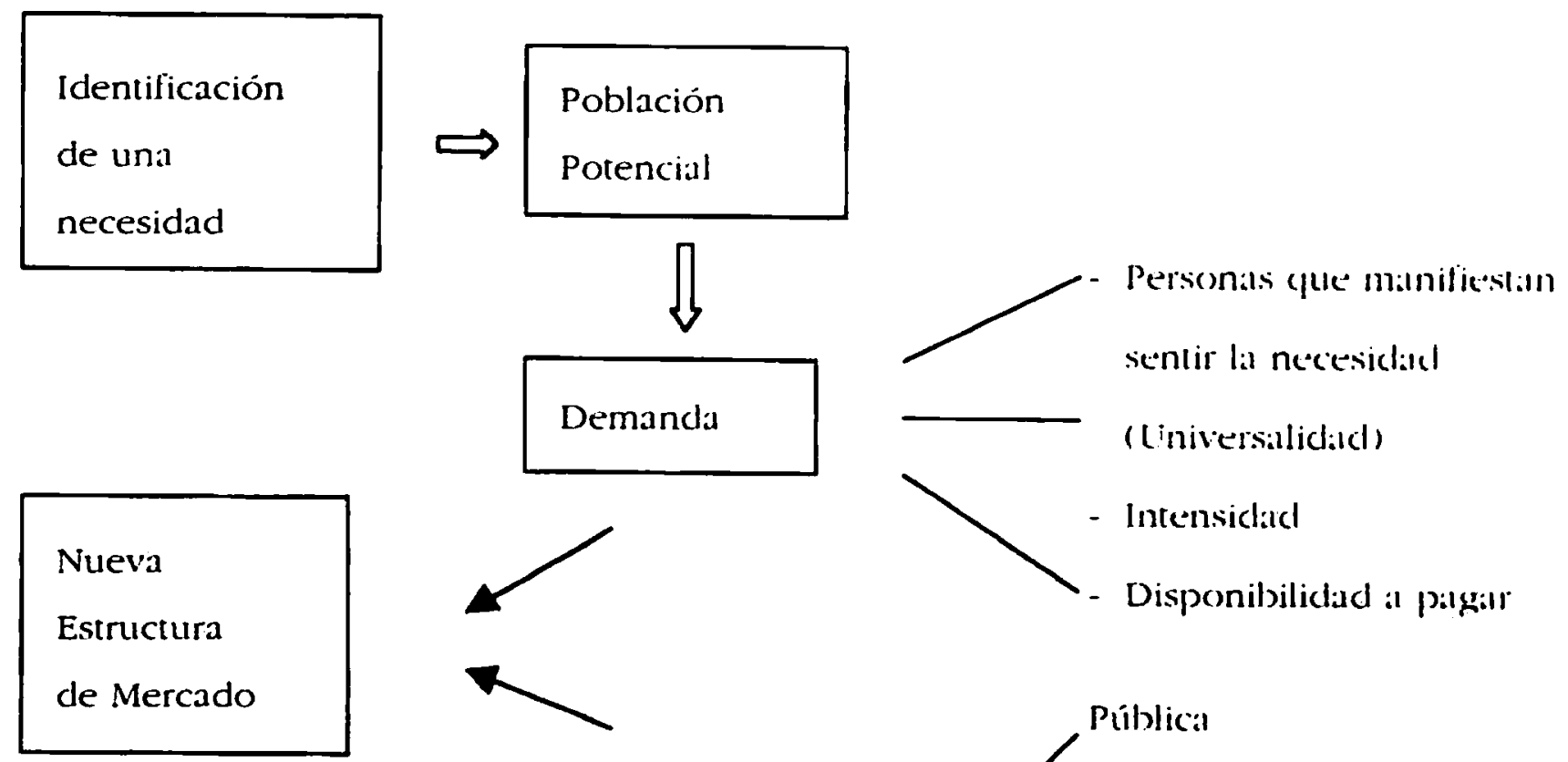

Ofertal
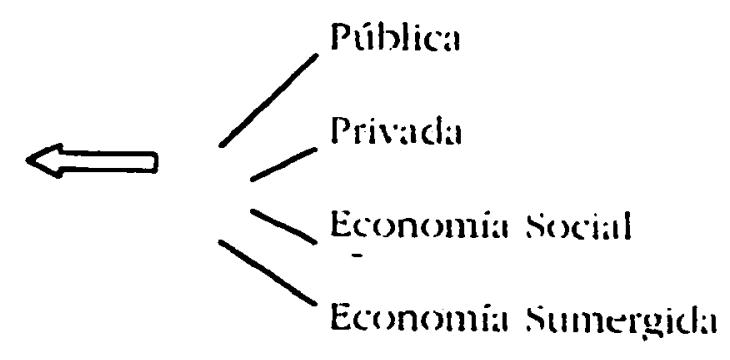

Coste indivisible

Intensiva en factor trabajo

Formación

Empleo 


\section{4. ÁmBito DE ACTUACIÓN}

El enfoque de los nuevos yacimientos de empleo implica una nueva concepción de la forma en que las autoridades pueden incidir en el mercado de trabajo para potenciar la creación de empleo. Las políticas macroeconómicas tradicionales de objetivos amplios e incidencia generalizada parecen haber perdido eficacia a medida que la sociedad contemporánea se ha vuelto más compleja y los sistemas económicos más volátiles. Por ello, se abre un campo de actuación más inmediato y cercano a los problemas cotidianos. En este sentido, el ámbito típico de actuación es el de las iniciativas locales, en un entorno geográfico concreto, como sería un distrito urbano, una zona rural o ciudades de pequeño y mediano tamaño.

Las iniciativas que se generan a partir de las nuevas fuentes de empleo se basan en nuevos principios de organización del tejido económico y social y dan protagonismo a los agentes de desarrollo del ámbito local.

En efecto, las iniciativas locales tienen grandes ventajas a la hora de implementarse. El buen conocimiento del medio y de la sociedad que las circunda permite a los agentes implicados la identificación de los yacimientos que pueden generar empleos de futuro en un entorno determinado".

La proximidad permite un mejor conocimiento de la oferta existente y favorece iniciativas en pro de la estructuración adecuada de la misma, una vez identificadas las necesidades sociales manifestadas. Así mismo. se plantea una mayor facilidad para concienciar a la población de referencia con vistas a transformar las necesidades manifestadas en demanda efectiva. Se trata, en todo caso, de aprovechar la diversidad territorial y las ventajas del espacio geográfico de referencia.

Las nuevas formas de intervención deben de aplicarse a todos los agentes con capacidad de proveer el bien o servicio por el que se manifiesta la necesidad. Así, a la iniciativa pública deben unirse tanto la iniciativa privada lucrativa como todos aquellos agentes que realizan su actividad dentro de la denominada economía social. Estos últimos agentes deben jugar un papel crucial en la estructuración de la oferta de estos bienes o servicios. Se trata de desocupación y el hecho de prescindir del afán de lucro posibilita el desarrollo de la actividad con un coste menor. En otro sentido, el voluntariado puede llevar aparejada también una mayor vocación, sobre todo en yacimientos integrados en ámbitos de una gran sensibilidad social (cuidado de ancianos, niños, medio ambiente,etc.).

La dinámica social promovida por las acciones referidas, debe redundar en una mejora de la economía local y sobre todo del bienestar de los ciudadanos que integran la comunidad de referencia.

6. Unión General de Trabajadores. El futuro del empleo y de la formación en la provincia de Sevilla. Cualificaciones profesionales con futuro y sus itinerarios, Sevilla, 1998. 
5. Los nuevos yacimientos y la creación de empleo en Andalucía. A modo de conclusión

Conocidos tanto la conceptualización como los elementos básicos que caracterizan las nuevas fuentes de empleo, es el momento de preguntarse si los nuevos yacimientos van a ser un factor importante para la generación de empleo en la economía andaluza.

Como hemos señalado. las características de las nuevas fuentes de empleo tienen que ver con el desarrollo económico y social, con la emergencia de las nuevas necesidades y con profundas transformaciones sociales y demográficas que configuran el entramado económico y social de la Europa de nuestros días.

Indudablemente, es posible percibir estos cambios en Andalucía, si bien el grado de desarrollo de los mismos debe matizarse dada la especial configuración de la región en términos económicos, sociales y culturales. La valoración de un yacimiento de empleo viene condicionada en buena medida por estos determinantes.

Podríamos señalar, en primer lugar, el menor nivel de renta andaluz si lo comparamos con el nivel medio español o europeo. El paso de necesidades a demanda implica una disponibilidad a pagar por parte de los ciudadanos, y en éste sentido, el factor renta es determinante. El diferencial de renta opera como una limitación que debe considerarse. Sobre todo si pensamos en la esfera privada como propulsora de la iniciativa, y dado el contexto de contención del gasto público en el que se mueve la economía europea, la iniciativa privada, lucrativa o social -que no significa deficitaria- parece la orientación más adecuada.

En segundo lugar, muchas de las actividades que se consideran como yacimientos de empleo carecen de un cálculo específico de costes, lo que afecta a la estructuración de la oferta potencial. En consecuencia se complica la evaluación de los puestos de trabajo a crear para la provisión del bien o servicio.

En tercer lugar, está el problema de la economía irregular. Las actividades de servicios que forman el grueso de las nuevas fuentes de empleo son el medio propio para desarrollar actividades al margen de la economía "oficial". Así, iniciativas como las descritas conseguirían cambiar cualitativamente el empleo, sacándolo a la superficie, pero no creándolo. En todo caso, la existencia de mercados paralelos o economía irregular para la provisión de determinados bienes o servicios pone de manifiesto la existencia de una demanda social para los mismos. Habría, no obstante, que señalar que el proceso de transformación de los empleos desde la economía irregular hasta la oficial supone un coste para las empresas que podria distorsionar la oferta final y naturalmente, vía precios, la demanda previamente existente. La naturaleza trabajo intensivo de muchos yacimientos dificulta aún más este proceso.

En cuarto lugar, el análisis de los nuevos yacimientos de empleo está íntimamente relacionado con un territorio y un sistema social que integra junto a los as- 
pectos económicos a los culturales y sociales. En el caso de Anclalucía, la estructura familiar juega aún un papel muy relevante como fuente última de aseguramiento y como provisión de muchos servicios de proximidad integrados en las nuevas fuentes de empleo. Aquí, hay que extremar la evaluación del potencial de empleo pues se trata de trasladar al mercado, servicios que se prestaban en unas condiciones de no mercado. (El cuidado de ancianos o de niños pequeños constituyen los casos más claros).

Debemos apuntar, por último, que el empleo creado en estas actividades, tendrá una remuneración asociada a su productividad, que dada su naturaleza intensiva en mano de obra tenderá a no ser muy alta. En este sentido, puede ahondarse en la dualización del mercado de trabajo, así como en una sociedad menos igualitaria ${ }^{7}$.

En estudios que hemos realizado aplicando la metodología antes mencionada se pone de manifiesto toda esta problemática y los resultados obtenidos han sido verdaderamente modestos. Además contrastan con los obtenidos en estudios similares en ciudades europeas como Marsella, en los que la estructura familiar y social son muy diferentes a las de Andalucía.

Los nuevos yacimientos constituyen una apuesta importante para la creación de empleo en una sociedad moderna. Sin embargo, consideramos que se está magnificando su potencialidad en un afán voluntarista de paliar un problema tan dramático como el desempleo. El peligro de plantearse objetivos previos en términos cuantitativos es evidente, pues puede distorsionar el verdadero calado del yacimiento.

Nuestra aproximación es general. Se precisarían costosos estudios de mercado para evaluar con precisión el potencial de cada yacimiento. Se necesitan estudios precisos para orientar esfuerzos y en este sentido, creemos que con el mejor manejo de datos disponibles y un análisis más riguroso del entorno se pueden conseguir evaluaciones próximas al estado real de la potencialidad del yacimiento.

\section{REFERENCLAS}

COLLADO CURIEL, J.C. y MARTÍNEZ MARTÍN, I (1995), "Generación de empleo a través de nuevos yacimientos: una simulación para el caso español". Economía y sociología del trabajo. № 29-30. Sept-Dic., pp. 24-34.

7. El último informe de la OCDE sobre perspectivas de empleo, 1997, publicado por el Ministerio de Trabajo y Asuntos Sociales, titula su editorial "los empleos de bajos salarios: eun trampolín hacia un futuro mejor o una trampa?", lo que constituye una clara llamada a la reflexión sobre estos temas.

8. Ver nota 5, PALMA et al. (1998a y 1998b). 
COLLÈGE COOPÉRATIF PROVENCE, Guide méthodologique pour la mise en ouvre d'une stratégie territoriale de création d'activités. Proyecto de Investigación financiado por la Dirección General V, de la Comisión de las Comunidades Europeas. Participan las Universidades de Génova, Patras y Sevilla, Junio 1998.

COMISIÓN DE LAS COMUNIDADES EUROPEAS (1993), Crecimiento, competitividad, empleo. Retos y pistas para entrar en el siglo XXI. Libro Blanco. Bruselas.

COMISIÓN DE LAS COMUNIDADES EUROPEAS (1996), Pacto de confianza. Acción a favor del empleo en Europa. Bruselas.

COMISIÓN EUROPEA (1995), Iniciativas locales de desarrollo y de empleo. Encuesta en la Unión Europea. Luxemburgo.

DÁVILA MURO, G. (1997), "El combate contra el desempleo en la Unión Europea: una estrategia común". Papeles de Economía Española", nº 72, pp. 294-308.

JIMÉNEZ, E; BARREIRO, F. y SÁNCHEZ, J.E. (1998), Los nuevos yacimientos de empleo. Los retos de la creación de empleo desde el territorio. Fundación Cirem. Barcelona.

JIMENO, J. F. (1998), "Aspectos determinantes del desempleo en España”. En El espacio social y el empleo en la construcción europea. pp. 54-55.

MARTÍN, C. (1997), España en la nueva Europa. Alianza. Madrid.

OCDE (1997), Perspectivas del empleo. 1997. Ministerio de Trabajo y Asuntos Sociales. Madrid, 1998.

PALMA, L y MARTÍN NAVARRO, J. L. (1998a), "Employment Policies: A methodology approach to the indentification of employment opportunities". $38^{b}$ Congress of the European Regional Science Association. 28 August-1 Septembre, 1998. Viena.

PALMA, L.; MARTÍN NAVARRO, J. L. y GARCÍA SANCHEZ, A. (1998b), "Una aproximación metodológica para la detección de oportunidades de empleo. Aplicación al servicio de asistencia domiciliaria a la tercera edad en las ciudades de Sevilla y Marsella". I Encuentro de Economía Aplicada. Barcelona.

TOHARÍA, L. (1998), El mercado de trabajo en España. McGraw Hill. Madrid.

UNIÓN GENERAL DE TRABAJADORES (1998). El futuro del empleo y de la formación en la provincia de sevilla, Cualificaciones profesionales con futuro $y$ sus itinerarios formativos. Sevilla. 